\title{
Risk Factors of Treatment Failure in Diabetic Foot Ulcer Patients
}

\author{
Kyung Mook Lee, Woon Hoe Kim, Jang Hyun Lee, Matthew Seung Suk Choi \\ Department of Plastic and Reconstructive Surgery, Hanyang University Guri Hospital, Hanyang University College of Medicine, Guri, Korea
}

Background Some diabetic feet heal without complication, but others undergo amputation due to progressive wounds. This study investigates the risk factors for amputation of diabetic feet. Methods A total of 55 patients who visited our institution from 2008 to 2012 were included in the study. The patients with abnormal fasting blood sugar levels, lower leg vascularity, and poor nutrition were excluded from the study group, and the wound states were unified. The patients were categorized into a treatment success group $(n=47)$ and a treatment failure group $(\mathrm{n}=8)$, and their hemoglobin $\mathrm{A} 1 \mathrm{C}(\mathrm{HgA} 1 \mathrm{C}), \mathrm{C}$-reactive protein (CRP), white blood cell count (WBC), and serum creatinine levels were analyzed.

Results The initial CRP, WBC, and serum creatinine levels in the treatment failure group were significantly higher than that of the treatment success group, and the initial $\mathrm{HgA} 1 \mathrm{C}$ level was significantly higher in the treatment success group. The CRP and WBC levels of both groups changed significantly as time passed, but their serum creatinine levels did not.

Conclusions The initial CRP, WBC, and serum creatinine levels were considered to be risk factors for amputation. Among them, the serum creatinine level was found to be the most important predictive risk factor. Because serum creatinine represents the renal function, thorough care is needed for the feet of diabetic patients with renal impairment.

Keywords Diabetes complications / Foot ulcer / Risk factors
Correspondence: Jang Hyun Lee Department of Plastic and Reconstructive Surgery, Hanyang University Guri Hospital, Hanyang University College of Medicine, 153 Gyeongchun-ro, Guri 471-701, Korea

Tel: +82-31-560-2330

Fax: +82-31-560-2338

E-mail: pslee1999@hanmail.net

This article was presented at the 2 nd Research and Reconstructive Forum on June 1-2, 2012 in Gwangju, Korea

No potential conflict of interest relevant to this article was reported.

\section{INTRODUCTION}

Type 2 diabetes mellitus (DM) is known to be a form of chronic metabolic syndrome, and its incidence rate has been increasing [1]. Due to the compliant use of insulin and the development of various oral hypoglycemic agents, the life expectancy of diabetic patients has been extended. With this trend, people have become more interested in the prevention and treatment of chronic rather than acute complications of DM. Examples of chronic DM complications include neuropathy, retinopathy, and diabetic foot ulcers. The incidence of diabetic foot ulcers is increasing at a higher rate, however, than that of the other complications [2].
In the case of diabetic foot ulcers, amputation must be considered if conservative treatment has failed. In the US, diabetic patients account for about $3 \%$ of the total population, and more than $50 \%$ of them undergo lower limb amputation [3]. About $1 \%$ to $4 \%$ of diabetic patients develop foot ulcers annually, and in $15 \%$, at least once in their lifetime [4]. Moreover, $85 \%$ of diabetic patients who have undergone lower limb amputations developed intractable diabetic foot ulcers [5]. Treatments available for diabetic foot ulcers range from conservative dressing to split- or full-thickness skin grafts, flap coverage, and major amputation, which is the last treatment option.

The worst complication in a patient with a diabetic foot is 
lower leg or foot amputation due to a non-healing wound with progressive infection. It is interesting, however, that even among wounds with similar initial states and which have been properly debrided, the treatment outcomes can differ greatly. The authors assessed hematologic risk factors such as hemoglobin A1C (HgA1C), white blood cell count (WBC), C-reactive protein (CRP), and serum creatinine because we observed that major limb amputation seemed to be influenced by the degree of diabetes, infection, and renal function. Many studies have reported that these risk factors are related to the treatment of diabetic foot ulcers; however, some sections of the results were controversial. Therefore, we analyzed these risk factors considering the severity of DM, ulcer wound, and vascularity.

\section{METHODS}

This study investigated the patients with diabetic feet who were hospitalized, visited the Outpatient Department, or consulted the Department of Endocrinology in the authors' institution from December 2008 to January 2012. The initial states of their wounds ranged from the first to third grades of the Wagner Grading Criteria (WGC). The patients consisted of 310 males and 92 females, with an average age of 65.4 years. This study was a retrospective study via a chart review. To exclude the effect of fasting blood sugar (FBS), 184 patients whose FBS level did not exceed 200 were selected, and among them, 116 patients without major posterior tibial artery and anterior tibial artery obstructions in their angiography were selected in order to rule out peripheral angiopathy-induced amputation. Of the 116 patients, 85 whose albumin level was more than $2 \mathrm{~g} / \mathrm{dL}$ were included, excluding those with delayed wound healing due to nutritional deficiency. This group was then reduced to 66 patients by only selecting those with third-WGC-grade ulcers. The final group of 55 patients was formed by excluding those who had experienced diabetic foot ulcers.

These 55 patients were categorized into a treatment success group and a treatment failure group. Their $\mathrm{HgA1C}$, WBC, CRP, and serum creatinine levels were compared and analyzed. Statistical analysis was performed using statistical software (SPSS ver. 14.0, SPSS Inc., Chicago, IL, USA), and the paired t-test and Wilcoxon signed rank test were used to determine statistically significant differences in the initial and late state of the same group. The Mann-Whitney $U$ test was used to determine statistically significant differences in the initial state of both groups. A P-value of less than 0.05 was considered statistically significant. The patients in the treatment success group experienced ulcer healing with conservative treatment such as antibiotic administration, regular dressing, use of a negative-pressure device, and/ or surgical methods such as local debridement, skin grafting, or a local or free flap. If the necrotic ulcer was limited to the toes, toe amputation was performed and the patient was included in the treatment success group. The treatment failure group included patients whose ulcers did not heal with the aforementioned procedures, and the wound state progressed to the fourth or fifth WGC grade, resulting in a major amputation such as an aboveknee, below-knee, Lisfranc, or ray amputation.

\section{RESULTS}

The treatment failure group consisted of 8 patients $(7$ males and 1 female): 5 underwent below-knee amputation, 1 underwent above-knee amputation, 1 underwent Lisfranc amputation, and 1 underwent Ray amputation (Fig. 1). Their mean age at the time of diagnosis with DM was 19 years, and the mean length of progression from the development of their initial wound to the fourth or fifth WGC grade was 55 days (range, 23 to 65 days). Their mean initial HgA1C, WBC, CRP, and serum creatinine levels were $9.7 \pm 2.4 \%, 13,462 \pm 2,769 / \mathrm{mm}^{3}, 6.8 \pm 2.6 \mathrm{mg} / \mathrm{dL}$, and $3.3 \pm 0.8 \mathrm{mg} / \mathrm{dL}$, respectively. These laboratory findings were rechecked 1 to 3 days before their scheduled major amputation, and the mean $\mathrm{HgA1C}, \mathrm{WBC}, \mathrm{CRP}$, and serum creatinine levels were $8.7 \pm 1.4 \%, 15,613 \pm 2,164 / \mathrm{mm}^{3}, 9.3 \pm 3.0 \mathrm{mg} / \mathrm{dL}$, and $3.4 \pm 2.5 \mathrm{mg} / \mathrm{dL}$, respectively. Only the WBC and CRP levels at a few days before major limb amputation were significantly higher than their initial levels $(\mathrm{P}=0.012$ and $\mathrm{P}=0.012$, respectively).

The treatment success group consisted of 47 patients ( 40 males and 7 females). Their mean age was 21.8 years at the time of diagnosis with DM, and the mean duration between initial treatment and wound healing was 38 days (range, 15 to 49 days).

\section{Fig. 1. A patient of the treatment failure group}

(A) The patient had acute renal failure, a diabetic ulcer on the lateral foot, and a bulla on the plantar area. (B) The wound was progressing, and septic conditions occurred. Below-knee amputation was performed to prevent the spread of infection.
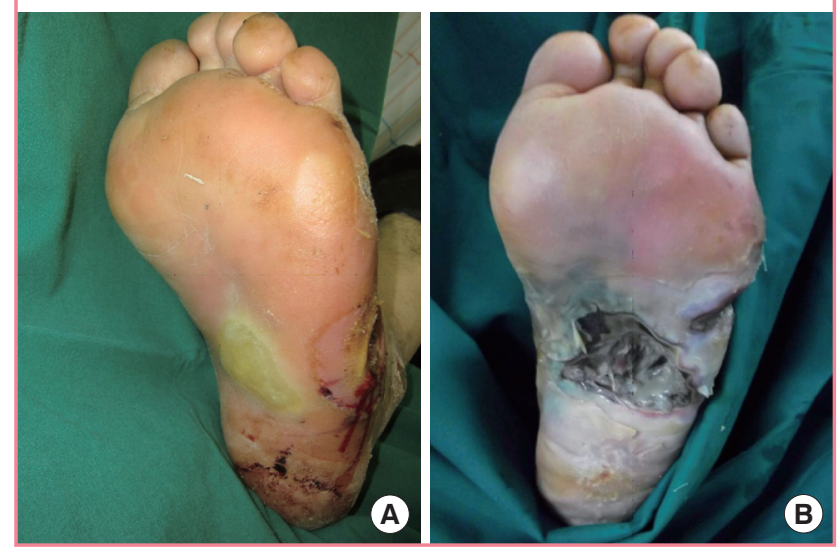
Healing was defined as the absence of any raw surface on the previous wound site or a wound state that approached the first WGC grade. In the treatment success group, 21 patients were treated with conservative measures such as the administration of appropriate antibiotics, regular topical dressing, and surgical debridement (Fig. 2). Of these, 9 patients underwent primary closure after adequate debridement, and 11 patients had splitthickness skin grafting on the wound beds of healthy granulation tissue after undergoing negative pressure therapy. Local and free flaps were performed in 4 and 2 patients with larger wounds, respectively (Fig. 3). All of the operations were performed when there were no signs of infection.

In the initial laboratory findings for the treatment success group, the mean $\mathrm{HgAlC}, \mathrm{WBC}, \mathrm{CRP}$, and serum creatinine levels were $10.6 \pm 1.5 \%, 10,179 \pm 2,911 / \mathrm{mm}^{3}, 3.1 \pm 4.6 \mathrm{mg} / \mathrm{dL}$, and $1.62 \pm 1.8$ $\mathrm{mg} / \mathrm{dL}$, respectively. After the wounds healed, these laboratory findings were rechecked. The mean $\mathrm{HgAlC}, \mathrm{WBC}, \mathrm{CRP}$, and serum creatinine levels were $10.5 \pm 1.5 \%, 8,460 \pm 1,447 / \mathrm{mm}^{3}$,

\section{Fig. 2. A patient of the treatment success group}

(A) The patient had a normal level of creatinine and had a necrotic ulcer on the first plantar metatarsal head area. (B) After appropriate debridement and conservative dressing, the wound was completely healed.
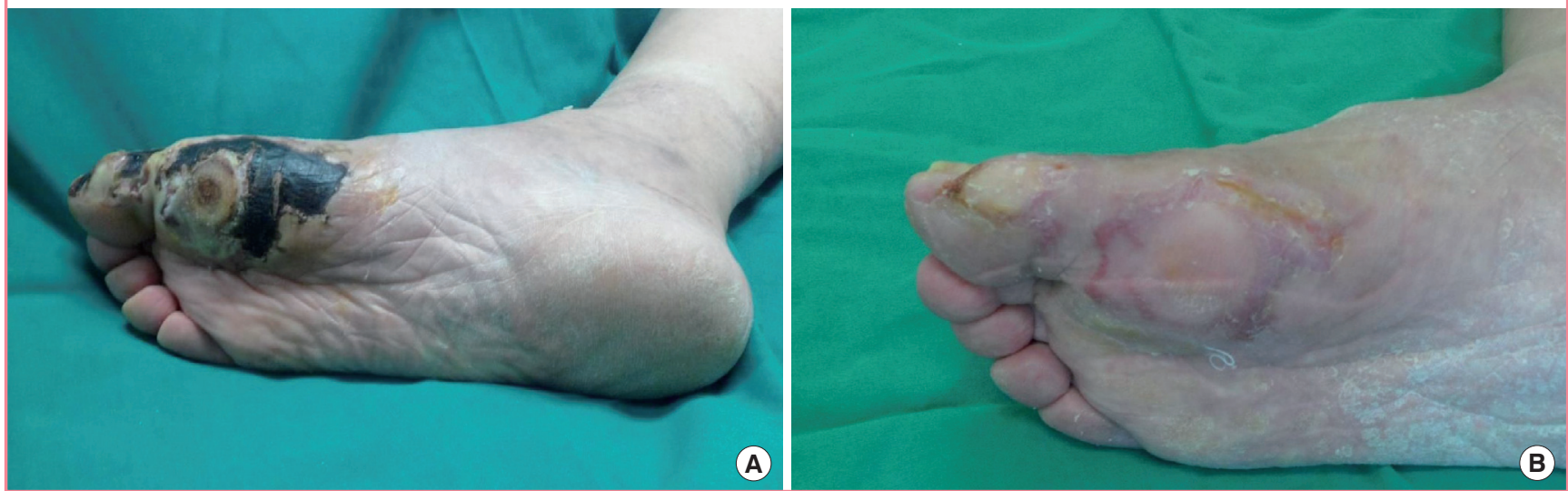

Fig. 3. A patient of the treatment success group

(A) The picture shows a skin and soft tissue defect on the right foot dorsum due to a diabetic ulcer. (B) The wound did not worsen beyond the foot dorsum, and we decided to treat it with anterolateral thigh fasciocutaneous free flap rather than amputation. (C) The picture shows the wound healing.
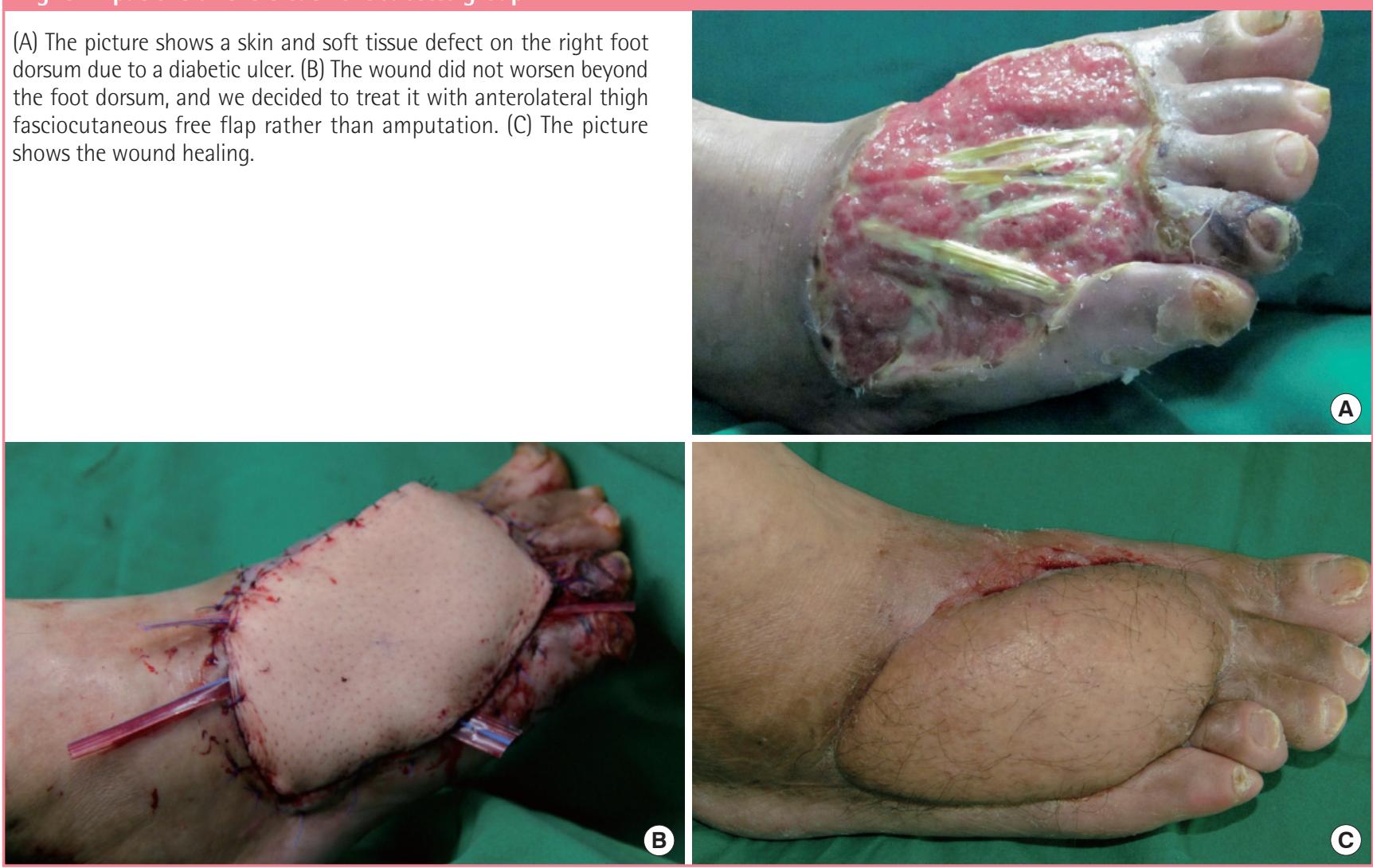
Fig. 4. HbA1C findings in the treatment success and failure groups

There was no difference in hemoglobin $\mathrm{A} 1 \mathrm{C}(\mathrm{HgA} 1 \mathrm{C})$, between the initialand late state in the two groups, and there was no difference between the initial state of the success and failure group.

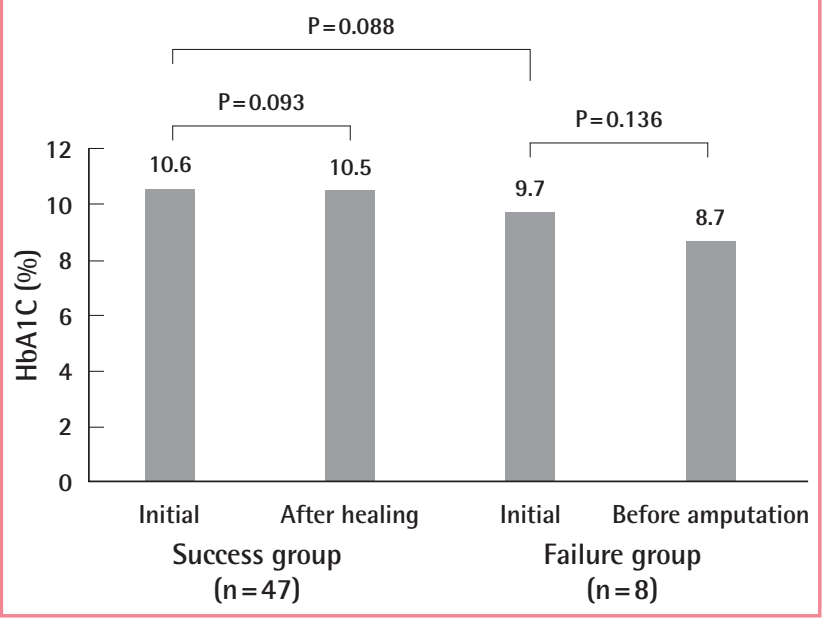

Fig. 5. WBC findings in the treatment success and failure groups

The white blood cell count (WBC) count was significantly lower in the late state than in the initial state of the success group, and it was significantly higher in the late state than in the initial state of the failure group. The WBC count was significantly higher in the initial state of the failure group than in the success group.

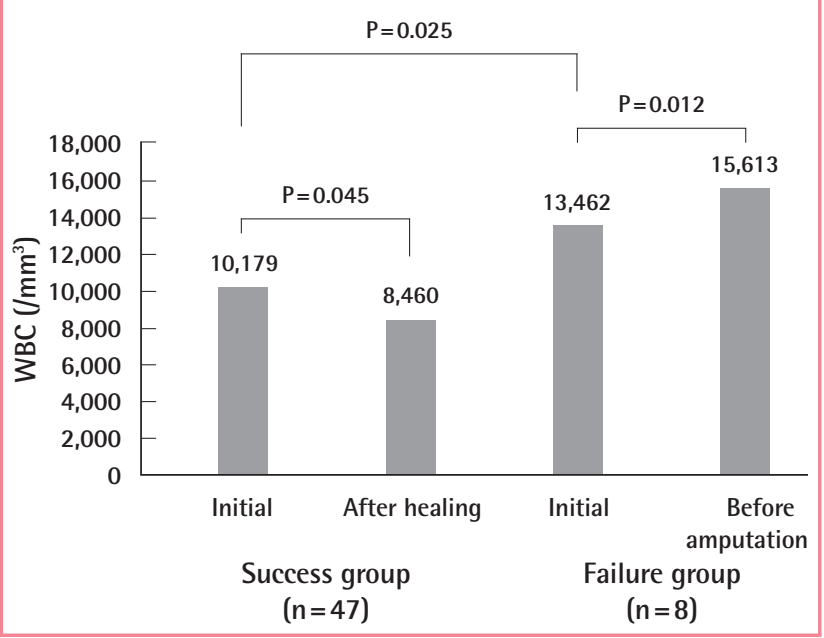

$1.5 \pm 1.2 \mathrm{mg} / \mathrm{dL}$, and $1.64 \pm 1.75 \mathrm{mg} / \mathrm{dL}$, respectively. These measurements were rechecked a mean of 29 days after the initial laboratory findings and showed a significant reduction in the WBC and CRP levels $(\mathrm{P}=0.045$ and $\mathrm{P}=0.001$, respectively).

In the evaluation of the risk factors based on the initial laboratory findings, the $\mathrm{HgAlC}$ level was higher in the treatment success group than in the failure group, but this finding was not statistically significant. On the other hand, the WBC, CRP, and serum creatinine levels were higher in the failure group than
Fig. 6. CRP findings in the treatment success and failure groups

C-reactive protein (CRP) was significantly lower in the late state than in the initial state of the success group, and it was significantly higher in the late state than in the initial state of the failure group. CRP was significantly higher in the initial state of the failure group than in that of the success group.

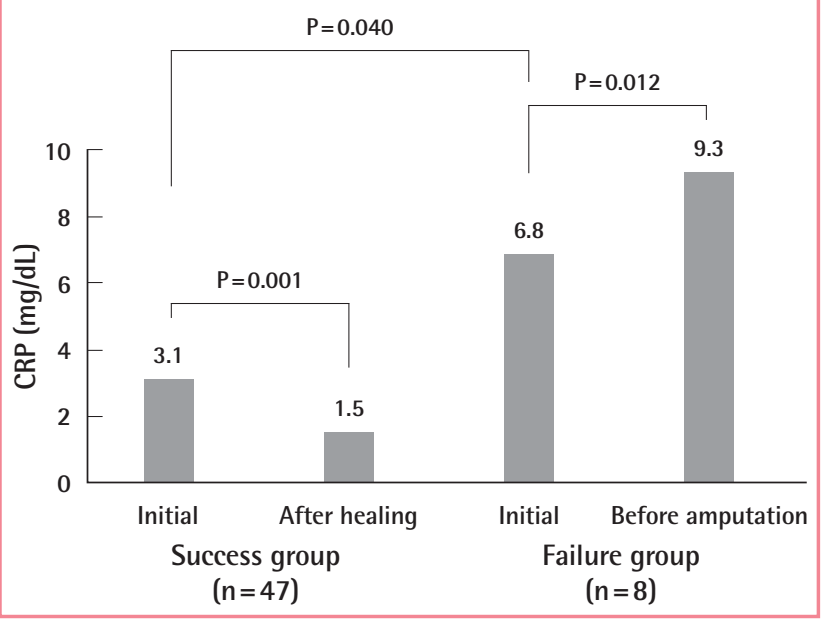

Fig. 7. Serum creatinine findings in the treatment success and failure groups

The serum creatinine levels showed no differences between the initial and late states in the two groups, but they were significantly higher in the initial state of the failure group than in that of the success group.

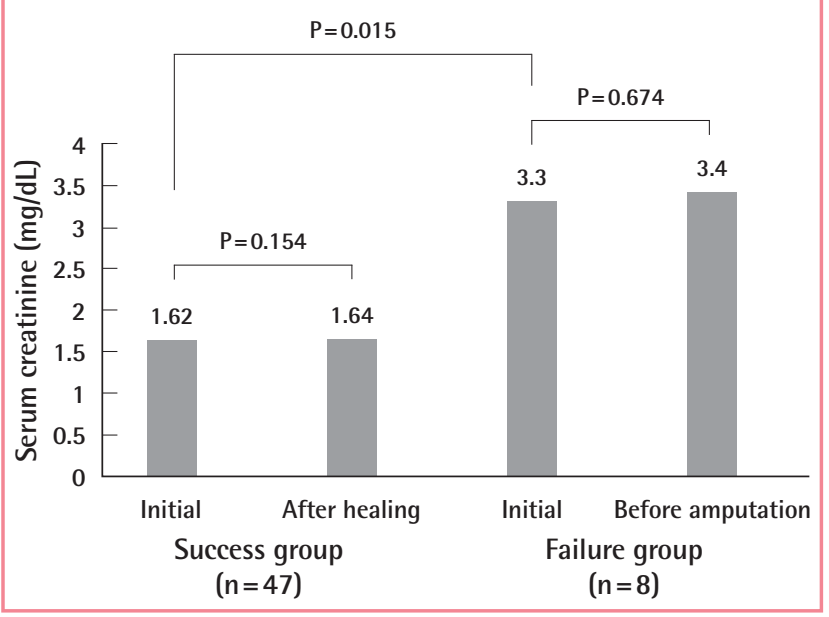

in the treatment success group. These results were statistically significant $(\mathrm{P}=0.025,0.04$, and 0.015 , respectively). The difference in the serum creatinine level had the most significant value (Figs. 4-7).

\section{DISCUSSION}

Many studies have been conducted to find the cause of diabetic foot disease and to evaluate the treatments of the disease. As mentioned, the main problem with diabetic foot disease is pro- 
gressive infection and a non-healing wound requiring amputation. Several doctors have carried out studies attempting to accurately predict and subsequently prevent this occurrence. Their experience shows that even among initially similar wounds, some healed with conservative treatment while others did not and eventually required amputation. As such, they concluded that there must be certain factors related to the failure of diabetic foot treatment. Sun et al. [6] reported that the Wagner classification had a remarkably close association with the potential risk factors of lower extremity amputation, showing different predictors with different grades. The traditionally recognized predictors of diabetic foot amputation, such as a lower ankle brachial index, albumin, or glomerular filtration rate, were almost exclusively found in the patients with Wagner grade 3 wounds, whereas in those with grades 2 and 4 wounds, the WBC count was identified as the primary predictor of lower extremity amputation [6]. Shojaiefard et al. [7] reported that nephropathy, peripheral vascular disease, and an initial FBS $>200 \mathrm{mg} / \mathrm{dL}$ were independent predictors of lower extremity amputation in patients hospitalized for diabetic foot lesions. Eneroth et al. [8] reported that an elevated WBC count reflected inflammation and infection of a diabetic foot, and is thus an important risk factor.

Some studies have reported that ethnicity was another risk factor. Yang et al. [9] reported that DM patients with renal disease and Malay ethnicity had higher rates of lower extremity amputation, compared to Indians and Chinese. Thus, Malay DM patients and diabetic patients with renal disease should be considered to be at high-risk for lower extremity amputation [9]. Karter et al. [10] reported that African-Americans had the highest risk factor of lower extremity amputation. Real Collado et al. [11] reported that diabetic patients with foot ulcers, a history of amputation, proliferative retinopathy, or osteomyelitis, and clinical signs of peripheral vasculopathy and/or severe infection had a high-risk of lower extremity amputation.

In a study by Sun et al. [6] and in the studies performedat the authors' institute, a higher $\mathrm{HbAlC}$ level has been shown to have less importance among the risk factors. However, the study by Resnick et al. [12] and Hennis et al. [13] reported a high $\mathrm{HbA1C}$ level to be a major risk factor of lower extremity amputation. Similarly, Sun et al. [6] did not identify renal function as a main risk factor; however, Margolis et al. [14], Yang et al. [9], and Akha et al. [15] did.

In our opinion, that these studies were designed with different methodologies and the selection of their study population was not randomized, explains their varying results. The authors found that patients who had lower extremity amputation were incapable of combating infection and had a high serum creatinine level. Thus, the authors investigated the correlation between diabetic foot treatment failure and the WBC and CRP levels, which reflects the presence and level of infection, and the serum creatinine level, which reflects the renal function. In addition, the authors compared the $\mathrm{HbAlC}$ levels to estimate the adequacy of diabetic control. Prior to the commencement of this study, the study subjects were selected among those who showed similar FBS levels, peripheral artery states, nutritional states, and initial wound grades in order to control the population and minimize bias. CRP has not been mentioned often, unlike WBC in other reports, but Jeandrot et al. [16] revealed that it is a major marker of diabetic foot infection.

The amputation group showed a low level of $\mathrm{HgAlC}$, which means it was not correlated strongly with diabetic foot treatment failure. On the other hand, WBC, CRP, and serum creatinine levels showed a high level of infection and disorder in renal function, which means they are the main risk factors predictive of diabetic foot treatment failure. In the comparison of the treatment failure group and the treatment success group, the serum creatinine level also did not significantly change between initial and late stage. In the treatment success group that had a low serum creatinine level in the initial stage, the CRP and WBC levels significantly decreased, whereas in the treatment failure group that had a high serum creatinine level, the data did not show a decrease, and no significant change was detected. With these results, the authors' institute assumed that if the serum creatinine level is low, the CRP and WBC levels can decrease even if they started at a high level, but if the serum creatinine level is high, the CRP and WBC levels cannot be controlled. This assumption can be supported by follow-up studies. Some studies have shown that a decrease in the renal function disturbs and inhibits neutrophil glycolysis [17]. If this renal function disorder intensifies and leads to the accumulation of uremic toxins in the body, it can cause phagocytic dysfunction and problems with infection control [18]. Chonchol [19] reported that the complication rate due to infection increased in patients with end stage renal disease. They also reported the mechanism of neutrophil dysfunction that can occur in renal impairment.

There have been some reports on the correlation between a high level of serum creatinine (nephropathy) and diabetic foot amputation $[15,20]$. However, they did not control the conditions of the patients. Amputation in diabetic patientsis caused by multifactorial events, and we believe that unification of other conditions is essential. Otherwise, it is possible that nephropathy or a decrease of renal function has less of a relationship with amputation. Our study has some limitations and problems. The study population size was small, and the controlled conditions were similar. However, if the controlled conditions were more diverse, the resulting population size would be even smaller, and 
this would have led to poor study results. The authors also did not factor in existing neuropathy in the study population, even though it is well-established that neuropathy can result in diabetic foot ulcers. The focus of this study, however, was on the hematological risk factors of lower limb amputation in diabetic foot patients; thus, the authors decided not to consider peripheral neuropathy. We excluded patients with major peripheral vascular disease (diagnosed via angiography), but included some patients with mild peripheral vascular disease (partial obstructions); therefore, the influence of partial obstructions on the treatment outcomes were not excluded. The last point that could have been improved is that gender differences and the age-dependent variance in serum creatinine level were not considered.

The major takeaways of this study are that the feet of diabetic patients must be thoroughly cared for, and that with improved renal function, the possibility of lower extremity amputation can be minimized. Speckman et al. [21] reported that inappropriate hemodialysis will increase the possibility of amputation; therefore, improving renal function and reducing the need for hemodialysis in DM patients can indirectly lead to a lower amputation rate. Again, thorough foot care and appropriate management of renal function are among the best ways of preventing lower extremity amputation in DM foot patients.

\section{REFERENCES}

1. Danaei G, Finucane MM, Lu Y, et al. National, regional, and global trends in fasting plasma glucose and diabetes prevalence since 1980: systematic analysis of health examination surveys and epidemiological studies with 370 country-years and 2.7 million participants. Lancet 2011;378:31-40.

2. Heo KB, Kim SM, Jang WI, et al. A clinical study in patients with diabetic gangrene. J Korean Diabetes Assoc 1983;7: 65-70.

3. Reiber GE, Boyko EJ, Smith DG. Lower extremity foot ulcers and amputation in individuals with diabetes. In: Harris MI, Cowie CC, Stern MP, et al., editors. Diabetes in America. 2nd ed. Washington, DC: National Institute of Diabetes and Digestive and Kidney Diseases; 1995. p. 408-28.

4. Reiber GE. Epidemiology of foot ulcers and amputations in the diabetic foot. In: Bowker JH, Pfeifer MA, editors. Levin and O'neal's the diabetic foot. St. Louis, MO: Mosby; 2001. p. 13-32.

5. Pecoraro RE, Reiber GE, Burgess EM. Pathways to diabetic limb amputation: basis for prevention. Diabetes Care 1990; 13:513-21.

6. Sun JH, Tsai JS, Huang CH, et al. Risk factors for lower extremity amputation in diabetic foot disease categorized by Wagner classification. Diabetes Res Clin Pract 2012;95: 358-63.

7. Shojaiefard A, Khorgami Z, Larijani B. Independent risk factors for amputation in diabetic foot. Int J Diabetes Dev Ctries 2008;28:32-7.

8. Eneroth M, Apelqvist J, Stenstrom A. Clinical characteristics and outcome in 223 diabetic patients with deep foot infections. Foot Ankle Int 1997;18:716-22.

9. Yang Y, Ostbye T, Tan SB, et al. Risk factors for lower extremity amputation among patients with diabetes in Singapore. J Diabetes Complications 2011;25:382-6.

10. Karter AJ, Ferrara A, Liu JY, et al. Ethnic disparities in diabetic complications in an insured population. JAMA 2002; 287:2519-27.

11. Real Collado JT, Valls M, Basanta Alario ML, et al. Factors associated with amputation in diabetic patients with foot ulcer. An Med Interna 2001;18:59-62.

12. Resnick HE, Carter EA, Sosenko JM, et al. Incidence of lower-extremity amputation in American Indians: the Strong Heart Study. Diabetes Care 2004;27:1885-91.

13. Hennis AJ, Fraser HS, Jonnalagadda R, et al. Explanations for the high risk of diabetes-related amputation in a Caribbean population of black african descent and potential for prevention. Diabetes Care 2004;27:2636-41.

14. Margolis DJ, Hofstad O, Feldman HI. Association between renal failure and foot ulcer or lower-extremity amputation in patients with diabetes. Diabetes Care 2008;31:1331-6.

15. Akha O, Kashi Z, Makhlough A. Correlation between amputation of diabetic foot and nephropathy. Iran J Kidney Dis 2010;4:27-31.

16. Jeandrot A, Richard JL, Combescure C, et al. Serum procalcitonin and $\mathrm{C}$-reactive protein concentrations to distinguish mildly infected from non-infected diabetic foot ulcers: a pilot study. Diabetologia 2008;51:347-52.

17. Vanholder R, Ringoir S, Dhondt A, et al. Phagocytosis in uremic and hemodialysis patients: a prospective and cross sectional study. Kidney Int 1991;39:320-7.

18. Ringoir S, Schoots A, Vanholder R. Uremic toxins. Kidney Int Suppl 1988;24:S4-9.

19. Chonchol M. Neutrophil dysfunction and infection risk in end-stage renal disease. Semin Dial 2006;19:291-6.

20. Young BA, Maynard C, Reiber G, et al. Effects of ethnicity and nephropathy on lower-extremity amputation risk among diabetic veterans. Diabetes Care 2003;26:495-501.

21. Speckman RA, Frankenfield DL, Roman SH, et al. Diabetes is the strongest risk factor for lower-extremity amputation in new hemodialysis patients. Diabetes Care 2004;27:2198-203. 